European Polymer Journal

Volume 90, May 2017, Pages 456-466

https://doi.org/10.1016/j.eurpolymj.2017.03.041

http://www.sciencedirect.com/science/article/pii/S0014305717300149

\title{
NATURAL ANTIOXIDANTS AS MELT STABILIZERS FOR PE: COMPARISON OF SILYMARIN AND QUERCETIN
}

Balázs Kirschweng ${ }^{1}$, Benedek Vörös ${ }^{1}$, Dóra Tátraaljai ${ }^{1,2}$, Miklós Zsuga $^{3}$, Enikö Földes ${ }^{1,2}$, Béla Pukánszky ${ }^{1,2}$

${ }^{1}$ Laboratory of Plastics and Rubber Technology, Department of Physical Chemistry and Materials Science, Budapest University of Technology and Economics, H-1521 Budapest, P.O. Box 91, Hungary

${ }^{2}$ Institute of Materials and Environmental Chemistry, Research Centre for Natural Sciences, Hungarian Academy of Sciences, H-1519 Budapest, P.O. Box 286, Hungary

${ }^{3}$ Department of Applied Chemistry, University of Debrecen, H-4002 Debrecen, P.O. Box 400, Hungary

*Corresponding author: Laboratory of Plastics and Rubber Technology, Department of Physical Chemistry and Materials Science, Budapest University of Technology and Economics, H-1521 Budapest, P.O. Box 91, Hungary. Tel: 36-1-463-2015, Fax: 36-1463-3474, E-mail: bpukanszky@ mail.bme.hu 


\section{ABSTRACT}

The stabilization effect of two flavonoid type natural antioxidants, silymarin ( $\mathrm{Si}$ ) and quercetin (Q), was compared in polyethylene (PE). Additive concentrations changed between 0 and 500 ppm in several steps and 1000 ppm Sandostab PEPQ phosphorus containing secondary stabilizer was also added to each compound. Stabilization efficiency was determined by changes in functional group (vinyl, residual PEPQ) content, melt flow rate (MFR), oxygen induction time (OIT), color and the consumption of the secondary antioxidant during multiple extrusions. The results showed that silymarin is a much less efficient stabilizer in polyethylene than quercetin. The consumption of vinyl groups is faster, melt flow rate and residual stability is smaller in its presence. Silymarin contains less active phenolic hydroxyls than quercetin, but comparison on equal molar basis also shows the inferiority of the compound. The difference can be partially explained by the larger bond dissociation enthalpies of the hydrogens in silymarin, but this antioxidant also accelerates the consumption of the phosphorous secondary stabilizer that must contribute to its smaller efficiency as well. DSC measurements indicate the interaction of the two compounds probably leading to the faster consumption of the phosphorous antioxidant and poor stabilization. Unlike quercetin and dihydromyricetin, the flavonoid type natural antioxidants studied earlier, silymarin is not a good candidate as stabilizer for practical applications.

KEYWORDS: polyethylene, processing stabilization, long chain branching, natural antioxidants, silymarin, 


\section{INTRODUCTION}

Plastics are exposed to various effects both during their processing and the application of the product that may result in their degradation. In order to prevent changes in their chemical structure, they contain stabilizers practically always. Polyethylene is routinely stabilized by the combination of a synthetic phenolic antioxidant and a secondary, processing stabilizer, usually a phosphorous compound. This simple stabilization package proved to be very efficient and cost effective thus the development of new stabilizers has not been in the focus of attention lately. Nevertheless, approximately a decade ago, some questions were raised about the potential health hazard generated by the reaction products formed from synthetic phenolic antioxidants during processing and use [1] and the questions have not been answered satisfactorily yet. The industry is not prepared with new solutions for the case if the fears about the harmful effect of phenolic antioxidants substantiated or regulations change dramatically.

Natural compounds offer an obvious solution to replace synthetic antioxidants. A large number of natural compounds proved to be beneficial for the human health because of their antioxidant, anti-inflammatory, antiviral or other effects and many of them are being used for therapy for a very long time. Some attempts have been made to use several natural compounds for the stabilization of polymers, and specifically polyolefins as well. Vitamin E proved to be a very efficient stabilizer [2-5] and it is presently used as antioxidant in ultrahigh molecular weight polyethylene implants [6-8]. Because of its polyaromatic structure and the number of phenolic hydroxyl groups in it, lignin is expected to have some stabilization effect in polymers, and several studies proved the assumption both in polyethylene and in polypropylene indeed [9-11]. A different compound, quercetin was also explored as a possible stabilizer both in food [12] and in polymers [13-14] and most of the results obtained indicated that it is a very efficient stabilizer. Dihydromyricetin, another 
flavonoid was also studied as stabilizer both in PE and PP and proved to be more efficient than the commercial stabilizers used as reference [15-16].

In recent years, our group set out to explore the possible range of natural compounds to be used as stabilizer in PE. The antioxidant effect of curcumin [17], quercetin [18] and dihydromyricetin [19] was studied in a Phillips type polyethylene in the presence of a phosphorous secondary stabilizer. All three proved to be very efficient, their stabilization efficiency exceed considerably that of the synthetic phenolic antioxidant used in the largest quantity in industrial practice. The stabilizers protected the polymer against degradation during processing already at the concentration of $50 \mathrm{ppm}$ and provided sufficient residual stability at $250 \mathrm{ppm}$. Another candidate in this line is silymarin, a flavonolignan compound with the same basic structure as quercetin, and it is used in therapy for more than two thousand years [20]. Silymarin is a unique flavonoid complex that is derived from the milk thistle plant and it contains, among others, silybin, silydianin, and silychristin [21]. The name comes from the traditional use of the substance by nursing mothers to increase milk, but it is best known for its use as a liver protectant and decongestant [22]. Similarly to other flavonoids, silymarin has antioxidant and antiviral effect [23-24], but it also increases glutathione levels and stimulates protein synthesis [25], thus the substance is a promising candidate for cancer therapy [26-30]. According to our knowledge, no attempt has been made to use silymarin as an antioxidant in polyethylene yet.

The goal of our work was to add another flavonoid type natural antioxidant, silymarin to the compounds studied up to now and explore the possibility of its use as antioxidant in PE. Just like in our previous works [17-19], silymarin was used in combination with a phosphorous secondary stabilizer to comply with industrial practice. The effect of the new antioxidant was compared to that of quercetin used as reference compound. The attention was focused first of all onto melt stabilization, but the effect of the 
two substances on residual stability and color was also determined. An attempt was made to explain similarities and differences based on the number, position and bond dissociation enthalpies of the phenolic hydroxyl groups located on the two compounds compared.

\section{EXPERIMENTAL}

\subsection{Materials}

The polymer used in the experiments was the Tipelin FS 471 grade ethylene/1hexene copolymer (melt flow rate: $0.3 \mathrm{~g} / 10 \mathrm{~min}$ at $190{ }^{\circ} \mathrm{C}, 2.16 \mathrm{~kg}$; nominal density: 0.947 $\mathrm{g} / \mathrm{cm}^{3}$ ) polymerized by a Phillips catalyst. The additive free polymer powder was provided by Tisza Chemical Ltd. (TVK), Hungary. Quercetin (95\%) was purchased from SigmaAldrich, while silymarin is curtesy of the Department of Applied Chemistry at the University of Debrecen [31]. To produce it, purple Silybum marianum seeds were collected in the region of Arad, Romania. 1200 grams of dried fruits were powdered, homogenized and defatted by hexane in a Soxhlet extractor for $6 \mathrm{~h}$. The resulting powder was dried and then macerated with acetonitrile, the solid was removed by sieving, then the solvent was evaporated and the crude silymarin was washed with ice-cold dichloromethane. The yield was $40 \mathrm{~g}$, which is $3.3 \mathrm{wt} \%$ of the initial quantity. The antioxidants were added to the polymer in various amounts, in 5, 10, 25, 50, 100, 250 and $500 \mathrm{ppm}$, to study the effect of additive content on stability. Each compound contained also 1000 ppm Sandostab PEPQ (PEPQ, Clariant) phosphonite secondary stabilizer.

\subsection{Sample preparation}

The polymer and the additives were homogenized in a high speed mixer (Henschel FM/A10) at a rate of $500 \mathrm{rpm}$ for $10 \mathrm{~min}$. The necessary amount of quercetin was dissolved in $200 \mathrm{~mL}$ acetone and the solution was added to the PE powder in the mixer. The resulting 
powder was dried overnight to remove acetone. Silymarin was added to the polymer together with PEPQ directly and homogenized in the high speed mixer under the same conditions as described above. The dry blend was processed and pelletized in six consecutive extrusion steps at $50 \mathrm{rpm}$ and barrel temperatures of $180,220,260$ and $260{ }^{\circ} \mathrm{C}$ under normal laboratory conditions using a Rheomex $\mathrm{S} 3 / 4$ " type single screw extruder attached to a Haake Rheocord EU 10V driving unit. Samples were taken after each extrusion step. For further studies, films of about $100 \mu \mathrm{m}$ thickness were compression molded at 190 ${ }^{\circ} \mathrm{C}$ and 5 min using a Fontijne SRA 100 machine.

\subsection{Characterization}

The melt flow rate (MFR) of the polymer was determined according to the ASTM D 1238-79 standard at $190{ }^{\circ} \mathrm{C}$ with $2.16 \mathrm{~kg}$ load using a Göttfert MPS-D MFR tester. Residual thermo-oxidative stability was characterized by the oxidation induction time (OIT) measured at $200{ }^{\circ} \mathrm{C}$ in oxygen atmosphere with constant, $20 \mathrm{ml} / \mathrm{min}$ flow rate in open aluminum pans using a Perkin Elmer DSC-7 apparatus. The concentration of the unsaturated functional groups of polyethylene were determined by FTIR spectroscopy on the $100 \mu \mathrm{m}$ thick compression molded films in transmission mode using a Tensor 27 (Bruker) spectrophotometer. Five parallel measurements were carried out on each sample between 4000 and $400 \mathrm{~cm}^{-1}$ wavelengths at $2 \mathrm{~cm}^{-1}$ resolution by 16 scans. Concentration of vinyl groups were calculated from the absorption at $908 \mathrm{~cm}^{-1}$. FTIR spectroscopy was used also for the determination of residual PEPQ content based on the absorption of P(III)-O-C groups at 850 $\mathrm{cm}^{-1}$. The color of the samples was described by the yellowness index (YI) and the optical $\mathrm{L}^{*}$ parameter measured on a Hunterlab Colourquest 45/0 apparatus. The thermal behavior of silymarin, that of its blends with PEPQ and component interactions were studied using a Perkin Elmer Diamond DSC-IC apparatus. Differential scanning calorimetric (DSC) 
measurements were carried out in nitrogen atmosphere with constant, $20 \mathrm{~mL} / \mathrm{min}$ flow rate in open aluminum pans, at a heating rate of $10{ }^{\circ} \mathrm{C} / \mathrm{min}$ from 0 to $300{ }^{\circ} \mathrm{C}$. In figures, silymarin is abbreviated as $\mathrm{Si}$, while quercetin as $\mathrm{Q}$ in order to increase clarity and help understanding.

\section{RESULTS AND DISCUSSION}

The results are discussed in several sections. First the composition and properties of silymarin are presented and then the main characteristics of the two natural antioxidants used in this study are compared to each other. The number of functional groups and reactivity are discussed in the next section. Processing stabilization, residual stability and color are presented in subsequent sections followed by the discussion of the results as well as some comments on practical consequences.

\subsection{Composition, properties}

Silymarin is not a single well defined compound, but a combination of various substances. The material extracted from milk thistle consists of two main components, 70$80 \%$ are flavonolignans and $20-30 \%$ fatty acids. The active component also contains several compounds, three main constituents and several minor ones, the latter being present in very small amounts. Silybin is the major component of the mixture being present at about $70 \%$. The composition of silymarin, the chemical structure of the components and their relative amounts are summarized in Table 1 [31]. 
Table 1 Average composition of the natural flavonolignan, silymarin [31]

Component

The comparison of chemical structures reveals the similarities, but also differences in the structure of the compounds and shows that they contain different number of phenolic hydroxyl groups at dissimilar positions. Obviously all are active compounds with stabilization activity, but their efficiency may differ considerably. In further discussion we assign the structure of silybin to the term silymarin. Although the other compounds react more efficiently with the DPPH ${ }^{\bullet}$ radical [32], their amount is much smaller in the mixture 
and the effect of silybin dominates. One might question the use of such a mixture as industrial additive, but the material is natural, extracted routinely and is available on the market.

The most important characteristics of the two compounds compared to each other are listed in Table 2. Quercetin which proved to be a very efficient melt stabilizer for PE had two drawbacks: its high melting temperature and strong yellow color. The melting temperature of silymarin is much smaller thus it melts during processing that must improve homogeneity. Unfortunately, the color of silymarin is also strong, but instead of yellow it is ocher. The chemical structure of the two compounds show some similarities and differences as well. The A and $\mathrm{C}$ rings are identical, but silymarin lacks the double bond between $\mathrm{C} 2$ and $\mathrm{C} 3$ carbon atoms. On the other hand the B ring is modified considerably. The number of active hydroxyls is also different as a result, while quercetin has five (the unsaturation in C ring increase conjugation as well as the activity of the hydroxyl group located at this ring), silymarin only three active hydroxyl groups. The difference in the number of reactive groups and also in the molecular weight of the two compounds must be taken into account during the evaluation of the efficiency of silymarin as stabilizer for PE. 
Table 2 Comparison of the characteristics of the two antioxidants used in the study

\begin{tabular}{|c|c|c|}
\hline Characteristics & Quercetin & Silymarin \\
\hline $\mathrm{M}_{\mathrm{n}}(\mathrm{g} / \mathrm{mol})$ & 302.2 & 482.4 \\
\hline $\begin{array}{l}\text { Melting point } \\
\left({ }^{\circ} \mathrm{C}\right)\end{array}$ & 316 & 167 \\
\hline Color & & \\
\hline Structure & & \\
\hline $\begin{array}{l}\text { Structural } \\
\text { differences }\end{array}$ & $\begin{array}{l}-\quad \text { double bond in } \mathrm{C} \text { ring } \\
-\quad 2 \text { hydroxyls in } \mathrm{B} \text { ring }\end{array}$ & $\begin{array}{ll}- & \text { single bond in } \mathrm{C} \text { ring } \\
- & 0 \text { hydroxyl in } \mathrm{B} \text { ring } \\
- & 1 \text { hydroxyl in } \mathrm{E} \text { ring }\end{array}$ \\
\hline $\begin{array}{l}\text { No. of phenolic } \\
\text { OH groups }\end{array}$ & 4 & 3 \\
\hline
\end{tabular}

Our previous study showed that in the determination of residual stability, the number of active hydroxyl groups was less important and the same residual stability was achieved with compounds containing different number of such groups [19]. Accordingly, although silymarin might be less efficient as stabilizer on a weight basis, it may prove to be effective in a comparison based on the number of active hydrogens.

\subsection{Functional groups, reactivity}

The reactivity and stabilization effect of phenolic antioxidants depend on their chemical structure, on the number of hydroxyl groups and their position. Four mechanisms 
have been proposed in the literature for the stabilization reactions of phenolic antioxidants: single electron transfer (SET) [33-34], sequential proton loss electron transfer (SPLET) [3536], radical adduct formation (RAF) [37] and hydrogen atom transfer (HAT) [38-39]. Because of the chemical character of the matrix and the radicals formed, the dominating mechanism is hydrogen atom transfer in polyethylene, but other reactions may also take place during processing. The rate of hydrogen transfer depends on the dissociation enthalpy of the hydrogen atom of the phenolic hydroxyl group.

Table 3 Bond dissociation enthalpies of active hydroxyl groups of the two flavonoid type natural antioxidants studied

\begin{tabular}{|c|c|c|}
\hline \multirow{2}{*}{ Active OH } & \multicolumn{2}{|c|}{ Bond dissociation enthalpy (kJ/mol) } \\
\cline { 2 - 3 } & Quercetin [40] & Silymarin [32] \\
\hline B4' & 305.0 & - \\
\hline B3' & 315.9 & 455.6 \\
\hline C3 & 339.5 & 400.0 \\
\hline A7 & 361.9 & 410.5 \\
\hline A5 & 398.3 & 367.8 \\
\hline E20 & - & 2 \\
\hline
\end{tabular}

Dissociation enthalpies can be predicted by quantum chemical calculations and they are listed in Table 3 for the two compounds compared here. Although the values were calculated by two different groups on different base sets, they offer some information about the expected reactivity of the two compounds. The B4' and B3' hydroxyls of quercetin have the smallest bond dissociation enthalpies thus they are expected to be the most active. These groups are absent in silymarin and even the hydroxyl group located in the $\mathrm{C} 3$ position lost 
its activity, because of the lack of double bond between the positions C2 and C3. The E20 hydroxyl is expected to be the most active in silymarin, but the bond dissociation enthalpy of even this hydroxyl is rather large.

Based on bond dissociation enthalpies, the activity of silymarin must be much smaller in stabilization reactions than that of quercetin. However, we must consider also the fact that the reactivity of hydroxyl groups changes after the first reaction, which may modify stabilizing efficiency completely. Moreover, the comparison of quercetin and dihydromyricetin clearly showed that in spite of the smaller dissociation enthalpy of the pirogallol moiety in DHM compared to that of the pyrocatechol structure in quercetin, the two compounds provided the same residual stability in PE [19]. Nevertheless, preliminary considerations predict smaller activity and less efficiency for silymarin than for quercetin.

\subsection{Processing stabilization}

The efficiency of the two antioxidants as melt stabilizers is characterized by the changes in the MFR of the polymer during multiple extrusions. MFR is plotted against the number of extrusions in Fig. 1 for compounds containing the two stabilizers in different amounts. We did not plot all the results in the figure, because the large number of points would make comparison very difficult, if not impossible. Moreover, the two stabilizers are not always compared at the same concentration, because of their very different efficiency. This latter statement is amply demonstrated by Fig. 1. 25 ppm quercetin has the same effect as $100 \mathrm{ppm}$ silymarin and the comparison of the two compounds at the largest concentration added also shows that silymarin is inferior to quercetin in melt stabilization as predicted by the comparison of bond dissociation enthalpies in section 3.2. 


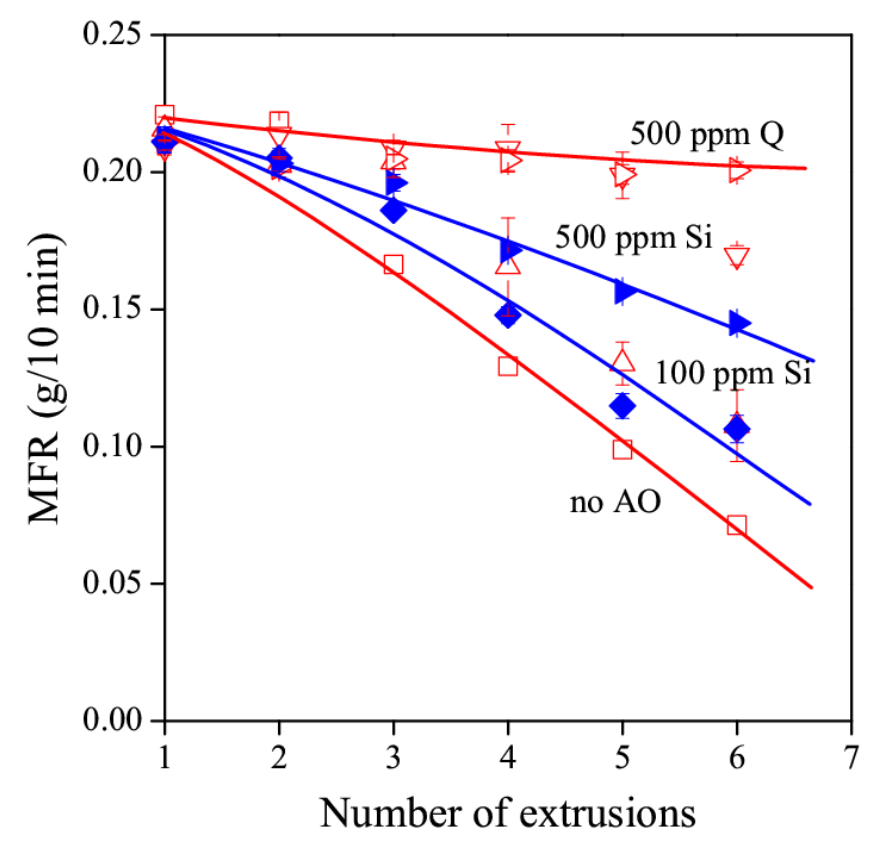

Fig. 1 Comparison of the melt stabilization efficiency of the natural antioxidants studied.

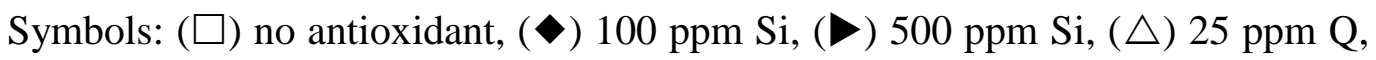

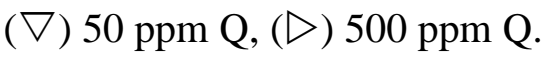

However, because of the dissimilar molecular weight of the compounds and their different number of active $\mathrm{OH}$ groups, comparison on a weight basis is misleading. Accordingly, MFR is plotted against the amount of natural antioxidant used in mmol active $\mathrm{OH} / \mathrm{kg}$ PE in Fig. 2 after the first and sixth extrusions in order to simplify the figure. The comparison shows that after the first extrusion, the effect of the two additives is very similar, but it differs considerably at longer processing history. Silymarin cannot protect the polymer against degradation after the sixth extrusion and the correlation indicates that even an increase in additive content would not result in better stability, in the prevention of the formation of long chain branches. The similarity after the first processing run needs further considerations and explanation. 


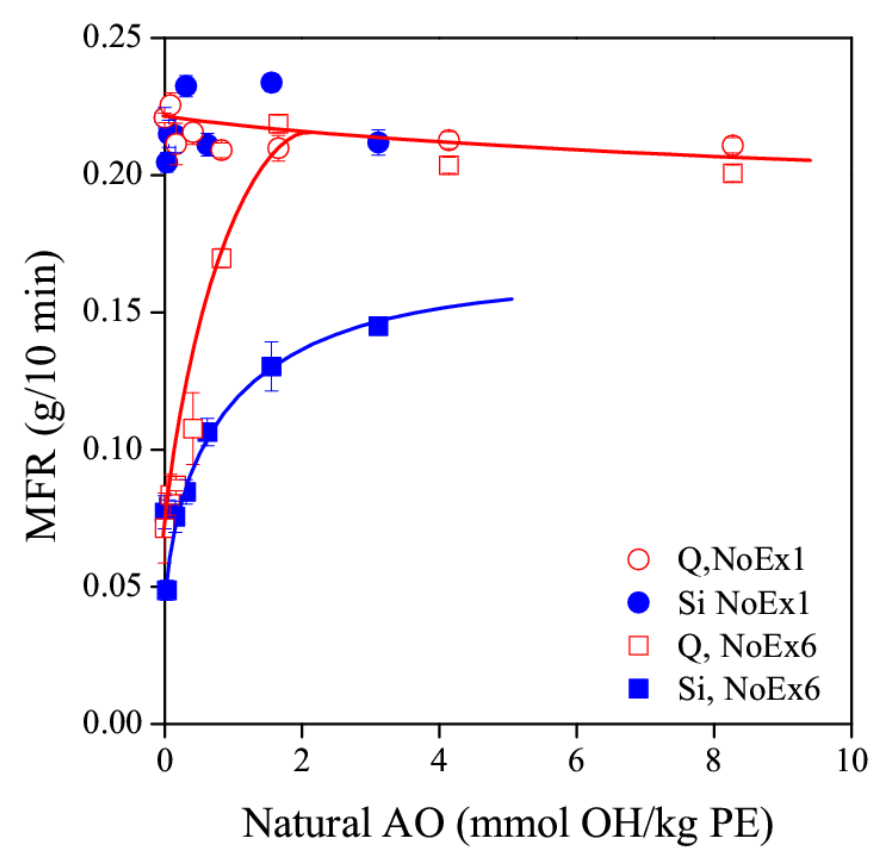

Fig. 2 Effect of the amount of natural antioxidant on the MFR of polyethylene at different processing histories. Symbols: (O) Si, NoEx1, (ם) Si, NoEx 6, (O) Q, NoEx1, ( $\square)$ Q, NoEx6.

The role of the secondary, phosphorous antioxidant in stabilization is crucial, especially in the first processing step. We showed in our previous study [19] that the larger efficiency of DHM resulted from the fact that it protected the phosphorous antioxidant better than quercetin during processing probably due to the interaction of the two compounds. Accordingly we may assume that the effect of silymarin on the reaction and consumption of the phosphorous antioxidant differs from that of quercetin. The residual amount of PEPQ is plotted against the number of extrusions in Fig. 3 . 


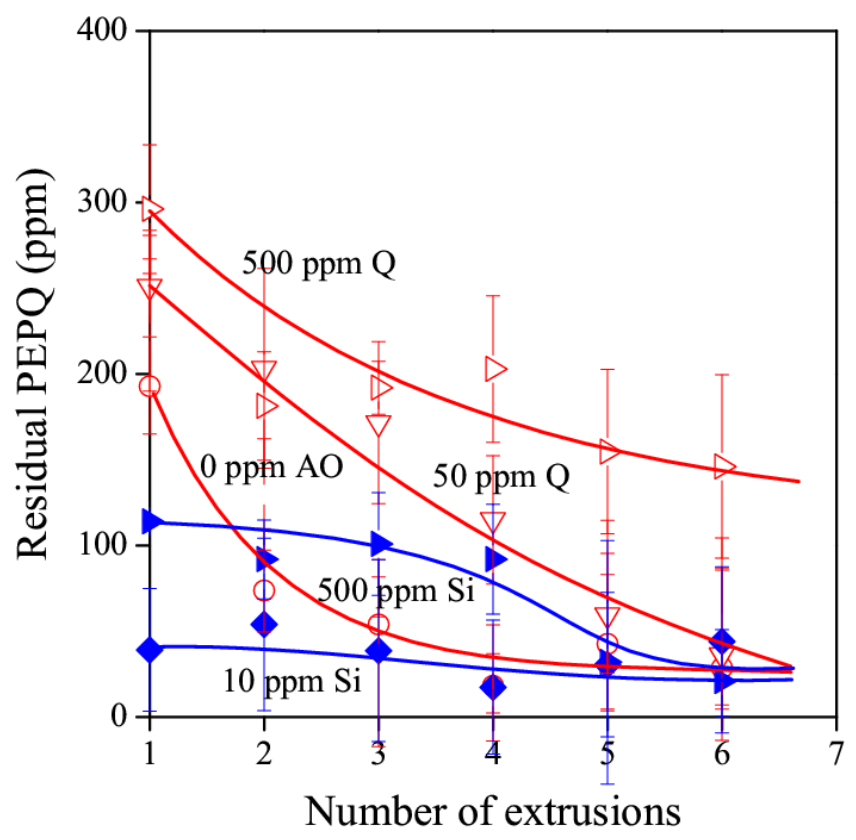

Fig. 3 Changes in the amount of residual phosphorous antioxidant with increasing number of extrusions. Symbols: $(\bigcirc)$ no antioxidant, $(\diamond) 10$ ppm Si, ( ) 500 ppm $\mathrm{Si},(\nabla) 50$ ppm Q, ( $) 500$ ppm Q.

The assumption presented above is completely justified. While quercetin protects the secondary stabilizer during processing and the amount of this latter is always larger when the combination of antioxidant/PEPQ is used than in the compound not containing the primary antioxidant. The situation is reversed in the presence of silymarin. Already $10 \mathrm{ppm}$ of silymarin decreases the level of PEPQ below that measured in the compound containing only the phosphorous antioxidant and after the first extrusion the concentration is quite small even at $500 \mathrm{ppm}$ silymarin content. We must emphasize here that the first extrusion is crucial, most of the chemical changes in the structure of the polymer take place at this stage, and the secondary stabilizer is the one that protects the polymer efficiently against such changes. Although at large silymarin content the consumption of PEPQ is slower than 
without it, PEPQ levels are still smaller than those achieved with the combination of PEPQ and $50 \mathrm{ppm}$ quercetin. The interactions between the primary and secondary stabilizers are obviously different when quercetin or silymarin are used and these interactions seem to play an important role in the determination of the efficiency of the additive package.

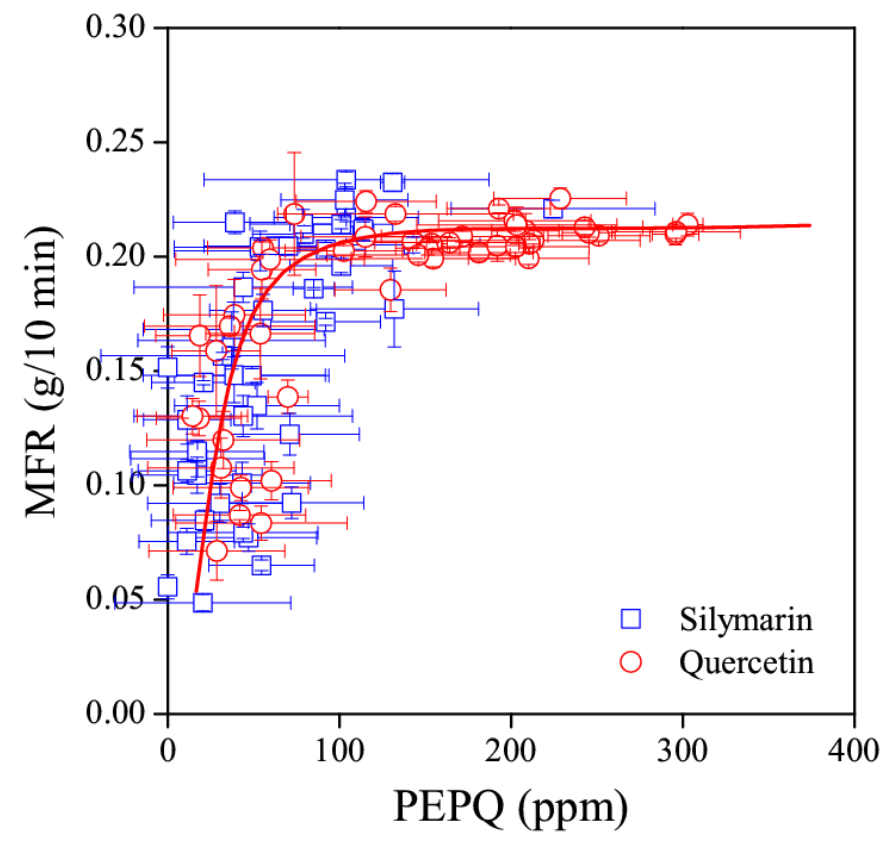

Fig. 4 Correlation between the melt viscosity (MFR) of the polymer and the residual amount of PEPQ in the compound. Symbols: $(\square)$ silymarin, $(\bigcirc)$ quercetin.

The crucial role of the primary antioxidant is clearly shown by Fig. 4 in which the melt flow rate of the polymer is plotted against its residual PEPQ content. The viscosity of the polymer remains constant until a certain level of PEPQ content is maintained, approximately at 75-100 ppm, but decreases drastically at smaller concentrations. The larger efficiency of quercetin is clearly shown by the fact that larger number of circles are located in the right hand side of the graph, while the opposite is valid for the left hand side, for smaller PEPQ contents. The interaction of the two compounds is certainly one of the main 
factors determining stabilizer efficiency and needs further study.

\subsection{Residual stability, color}

Some products, like gas and water pipes, must possess sufficient residual stability in order to reach the necessary lifetime and this must be provided by the stabilizer package. As a consequence, primary stabilizers must be efficient and added in the necessary amounts. The residual stability characterized by the oxygen induction time is plotted against the number of extrusions in Fig. 5. All the data were plotted in the figure this time. According to the results 250 or $500 \mathrm{ppm}$ quercetin are sufficient to achieve adequate residual stability, but all the rest of the compounds have OIT values smaller than $10 \mathrm{~min}$, including the one containing 500 ppm silymarin. Obviously, this latter natural antioxidant cannot protect the polymer during long term use and cannot be applied in practice. 


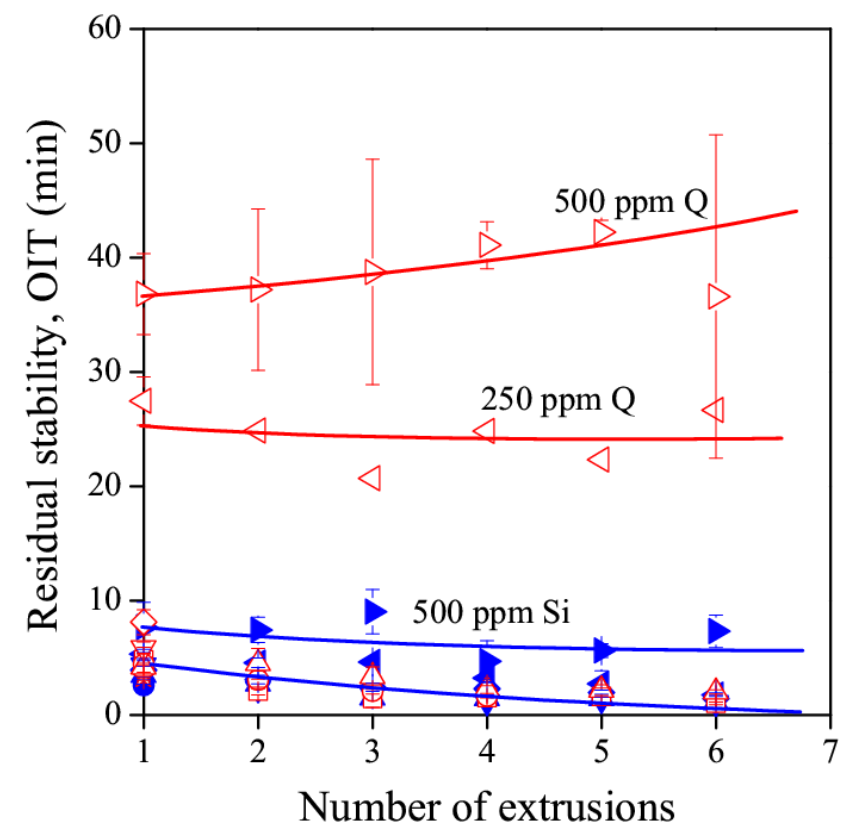

Fig. 5 Residual stability (OIT) of polyethylene containing in various amounts the natural

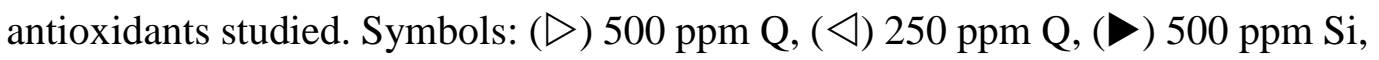
the rest of the series cannot be distinguished thus the corresponding symbols are not given here. See compositions in the experimental part.

However, comparison is biased again by the dissimilar number of active hydroxyl groups and by the different molecular weight of the compounds studied. Residual stability is plotted against the concentration of the natural antioxidants expressed in mmol OH/kg PE in Fig. 6. Residual stability changes linearly with increasing amount of phenolic antioxidant as shown by several literature references [41-43], but the slope of the change differs considerably for the two compounds. Obviously, the active hydrogens in silymarin are less efficient than those in quercetin and the different efficiency is probably the consequence of dissimilar bond dissociation enthalpies. One might wonder about the similar stabilities after the first and the sixth extrusion, but this effect was explained earlier with the limited 
solubility of these compounds in polyethylene and the dissolution of the stabilizer from the dispersed, heterogeneous stabilizer particles during subsequent extrusions [18]. Although the solubility of silymarin is somewhat larger on weight basis, around $45 \mathrm{ppm}$ compared to the $20 \mathrm{ppm}$ for quercetin, but it is practically the same in mols. OIT results confirm the conclusions drawn from the study of MFR and show that the efficiency of silymarin is considerably smaller than that of quercetin.

The strong color of most natural antioxidants might be regarded an obstacle before their application in certain areas. Quercetin was shown to discolor the polymer considerably. The yellowness index of the polymer is plotted against additive content in Fig. 7. The yellow color of the polymer containing quercetin is very strong and similar after the first and sixth extrusions. In fact a more thorough scrutiny reveals that color decreases with increasing number of extrusions which is quite unusual. The yellow color is caused by the stabilizer itself, its degradation products are less colored thus the color of polyethylene decreases with increasing number of extrusions because of the consumption of the additive. Table 2 showed that silymarin is ocher thus it also discolors the polymer. However, the color obtained is somewhat less intense than that with quercetin and it increases with increasing number of extrusion indicating that reaction products are more colored. In spite of the smaller intensity, the color of PE containing silymarin is still quite strong for applications in which colorless or white product is needed. Fortunately, many products are dark or even black in practical applications. 


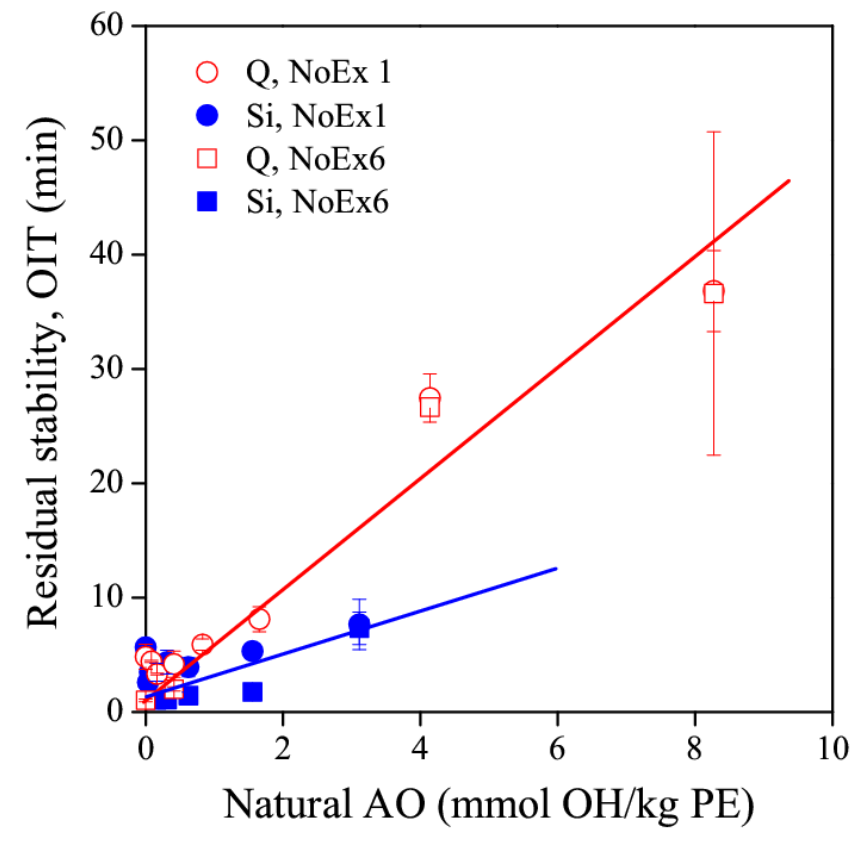

Fig. 6 Comparison of the effect of the two antioxidants on the residual stability of PE. The comparison is done on equal functional group content. Symbols are the same as in Fig. 2. 


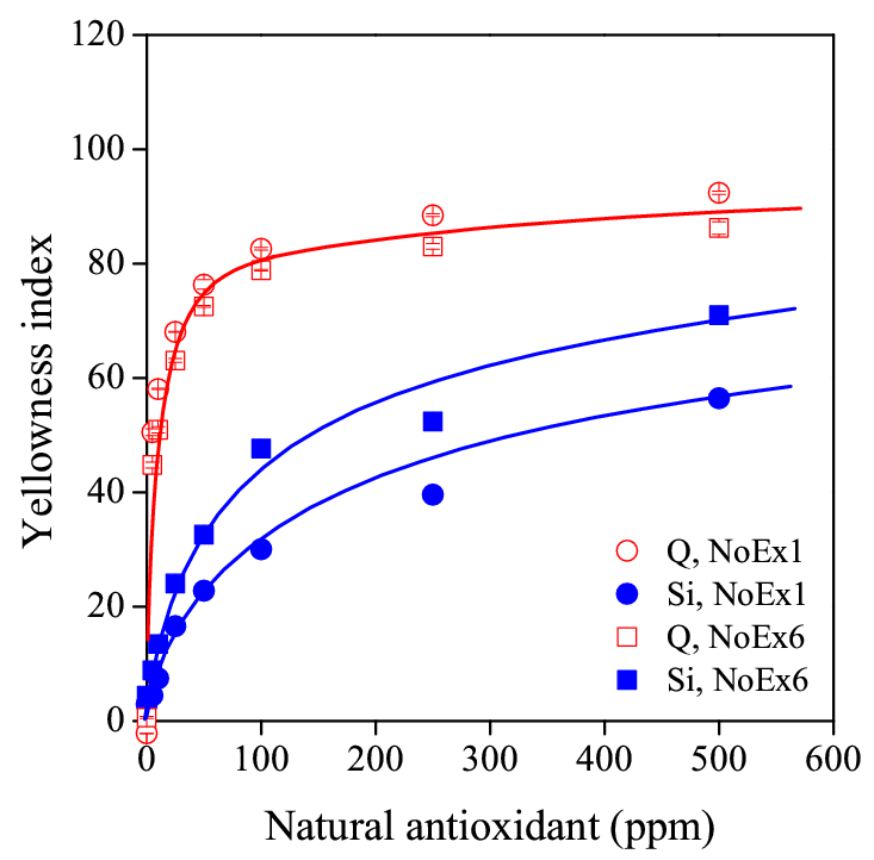

Fig. 7 Effect of the amount of natural antioxidants and the number of extrusions on the color of PE. Symbols are the same as in Fig. 2.

\subsection{Discussion, interactions}

The results obtained clearly show that silymarin is a less efficient natural antioxidant than quercetin. The consumption of vinyl groups is much faster in the presence of silymarin than with quercetin (Fig. 8) indicating the formation of long chain branches. As a consequence MFR decreases faster and also the residual stability of the polymer containing silymarin is inferior. The comparison of the two compounds on the basis of equal number of active hydroxyl groups indicated that the smaller efficiency does not result from the smaller number of reactive groups, but probably from the larger bond dissociation enthalpy of the hydrogens on the phenolic $\mathrm{OH}$ groups. All these result indicate that silymarin is not a good candidate for practical use as stabilizer in polyethylene. 


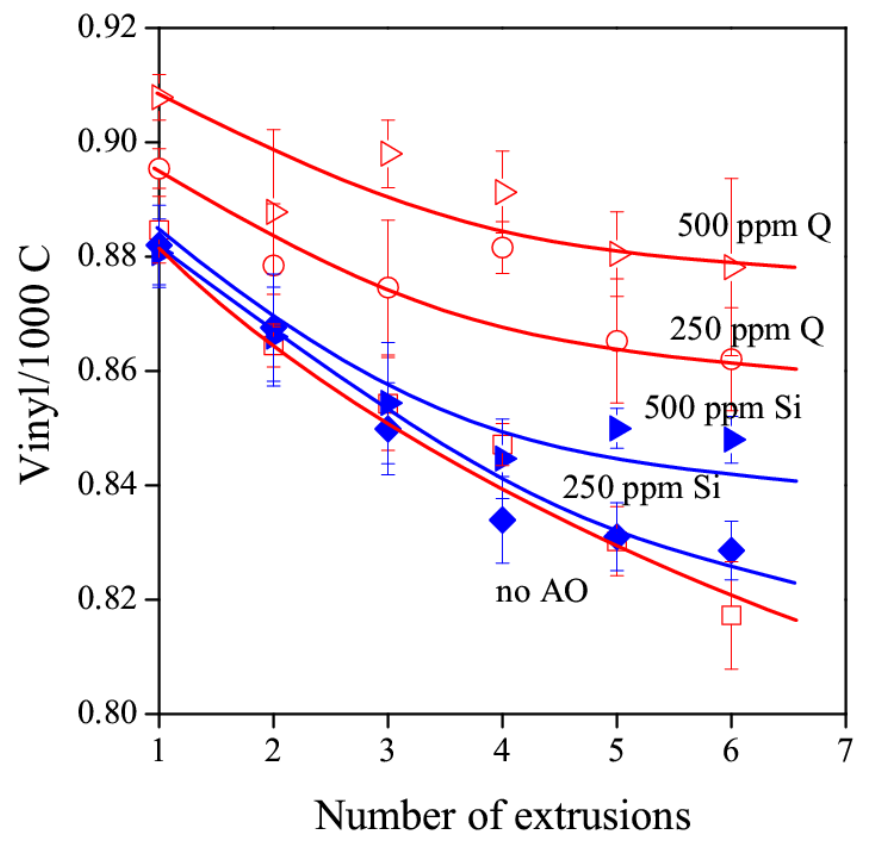

Fig. 8 Changes in the vinyl group content of polyethylene as a function of the processing history. Symbols: $(\square)$ no antioxidant, ( ) 250 ppm Si, ( $) 500$ ppm Si, (○) 250 ppm Q, ( $\triangleright 500$ ppm Q.

However, the measurement of residual PEPQ content also showed clear differences between the two natural antioxidants. Quercetin protected the phosphorous compound, its consumption decreased with increasing amounts of the flavonoid. On the other hand, the rate of PEPQ consumption increased in the presence of silymarin that might have also contributed to the smaller efficiency of this additive. The differences in PEPQ consumption cannot be explained with the dissimilar bond dissociation enthalpies of the hydrogens in the two compounds. We assume that the different rate of PEPQ consumption is the result of the interaction of the primary and secondary antioxidants, which probably differs in the two cases. We proved the existence of such interactions earlier by changes in the DSC traces of the combination of natural antioxidants and PEPQ [18-19]. We carried out similar 
measurements for silymarin as well, and a few traces are presented in Fig. 9.

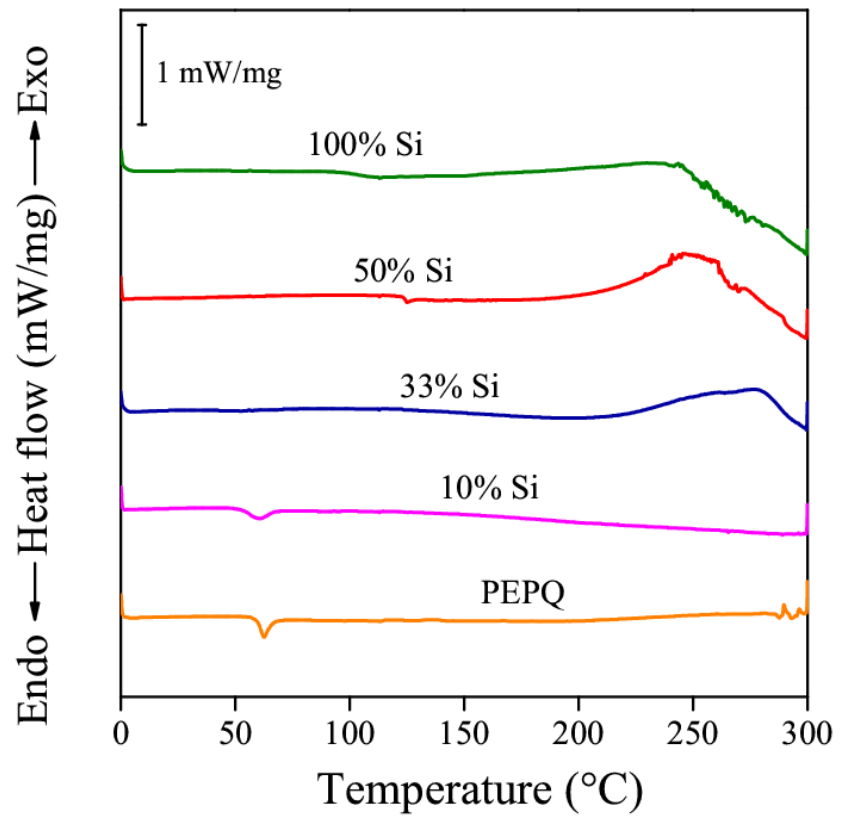

Fig. 9 DSC traces of silymarin, PEPQ and mixtures of the two recorded in the first heating run. Effect of interactions on melting (PEPQ) and decomposition (Si).

According to the traces the small melting peak of PEPQ disappears already at $30 \%$ silymarin content and the decomposition of this latter starts at higher temperature with increasing PEPQ content. Both changes indicate indeed that the two compounds interact with each other. However, we do not know anything about the nature of these interaction. The consumption of the secondary, phosphorous antioxidant must be related to these interactions, since it was the slowest in the presence of dihydromyricetin, somewhat faster with quercetin, but still protecting the secondary antioxidant, while consumption was very fast in the presence of silymarin, which accelerated it. The melt stabilization efficiency of the three compounds changed accordingly, the largest was for DHM and the smallest for silymarin. It is safe to assume that the two processes, i.e. PEPQ consumption and melt 
stabilization, are related and worth more attention, considerations and study in the future.

\section{CONCLUSIONS}

The comparison of two natural antioxidants, silymarin and quercetin used as reference showed that silymarin is a much less efficient stabilizer in polyethylene than quercetin. The consumption of vinyl groups is faster and melt flow rate as well as residual stability is smaller in its presence. Silymarin contains less active hydroxyls than quercetin, but comparison on equal molar basis also shows the inferiority of the compound. The difference can be partially explained by the larger bond dissociation enthalpies of the hydrogens in silymarin, but silymarin also accelerates the consumption of the phosphorous secondary stabilizer that must contribute to its inferior efficiency as well. DSC measurements indicate the interaction of the two compounds probably leading to the faster consumption of the phosphorous antioxidant and poor stabilization. Unlike quercetin and dihydromyricetin, the flavonoid type natural antioxidants studied earlier, silymarin is not a good candidate as stabilizer for practical applications.

\section{ACKNOWLEDGEMENTS}

The National Research Fund of Hungary (OTKA K 120039) is acknowledged for the financial support of the research.

\section{REFERENCES}

[1] Brocca D, Arvin E, Mosbaek H. Identification of organic compounds migrating from polyethylene pipelines into drinking water. Water Res 2002;36(15):3675-3680.

[2] Al-Malaika S, Ashley H, Issenhuth S. The antioxidant role of $\alpha$-tocopherol in polymers. I. The nature of transformation products of $\alpha$-tocopherol formed during melt 
processing of LDPE. J Polym Sci A-1 1994;32:3099-3113.

[3] Al-Malaika S, Goodwin C, Issenhuth S, Burdick D. The antioxidant role of alphatocopherol in polymers II. Melt stabilising effect in polypropylene. Polym Degrad Stab 1999;64(1):145-156.

[4] Al-Malaika S, Issenhuth S. The antioxidant role of alpha-tocopherol in polymers III. Nature of transformation products during polyolefins extrusion. Polym Degrad Stab 1999;65(1):143-151.

[5] Al-Malaika S, Issenhuth S, Burdick D. The antioxidant role of vitamin E in polymers V. Separation of stereoisomers and characterisation of other oxidation products of DLalpha-tocopherol formed in polyolefins during melt processing. Polym Degrad Stab 2001;73(3):491-503.

[6] Delfosse D, Lerf R, Zurbru D, Ag N, Zu E. Use of vitamin E to protect cross-linked UHMWPE from oxidation. Biomaterials 2010;31:3643-3648.

[7] Shen J, Costa L, Xu Y, Cong Y, Cheng Y, Liu X, Fu J. Stabilization of highly crosslinked ultra high molecular weight polyethylene with natural polyphenols. Polym Degrad Stab 2014;105:197-205.

[8] Affatato S, Salvatore J, Mattia D, Bracco P, Pavoni E, Taddei P. Wear performance of neat and vitamin E blended highly cross-linked PE under severe conditions: The combined effect of accelerated ageing and third body particles during wear test. $\mathbf{J}$ Mech Behav Biomed Mater, 2016;64:240-252.

[9] Alexy P, Kosikova B, Podstranska G. The effect of blending lignin with polyethylene and polypropylene on physical properties. Polymer 2000;41(13):4901-4908.

[10] Pouteau C, Dole P, Cathala B, Averous L, Boquillon N. Antioxidant properties of lignin in polypropylene. Polym Degrad Stab 2003;81(1):9-18.

[11] Gregorová A, Kosikova B, Moravcik R. Stabilization effect of lignin in natural rubber. 
Polym Degrad Stab 2006;91(2):229-233.

[12] Shahidi F, Ambigaipalan P. Phenolics and polyphenolics in foods, beverages and spices : Antioxidant activity and health effects. J Functional Foods 2015;18:820-897.

[13] Koontz JL, Marcy JE, O'Keefe SF, Duncan SE, Long TE, Moffitt RD. Polymer processing and characterization of LLDPE films loaded with alpha-tocopherol, quercetin, and their cyclodextrin inclusion complexes. J Appl Polym Sci 2010;117(4):2299-2309.

[14] Samper MD, Fages E, Fenollar O, Boronat T, Balart R. The potential of flavonoids as natural antioxidants and UV light stabilizers for polypropylene. J Appl Polym Sci 2013;129(4):1707-1716.

[15] Xin M, Ma Y, Xu K, Chen M. Dihydromyricetin: An effective non-hindered phenol antioxidant for linear low-density polyethylene stabilisation. J Therm Anal Calorim 2013;114(3):1167-1175

[16] Xin M, Ma Y, Lin W, Xu K, Chen M. Use of dihydromyricetin as antioxidant for polypropylene stabilization. J Therm Anal Calorim. 2015;120(3):1741-1747.

[17] Tátraaljai D, Kirschweng B, Kovács J, Földes E, Pukánszky B. Processing stabilisation of PE with a natural antioxidant, curcumin. Eur Polym J 2013;49(6):1196-1203.

[18] Tátraaljai D, Földes E, Pukánszky B. Efficient melt stabilization of polyethylene with quercetin, a flavonoid type natural antioxidant. Polym Degrad Stab 2014;102(1):4148.

[19] Kirschweng B, Bencze K, Sárközi M, Hégely B, Samu Gy, Hári J, Tátraaljai D, Földes E, Kállay M, Pukánszky B. Melt stabilization of polyethylene with dihydromyricetin , a natural antioxidant. Polym Degrad Stab 2016(11);133:192-200.

[20] Morazzoni P, Bombardelli E. Silybum marianum (Carduus marianus). Fitoterapia 
$1995 ; 64: 3-42$.

[21] Šimanek V, Křen V, Ulrichova J, Vičar J, Cvak L L. Silymarin: What is in the name...? An appeal for a change of editorial policy. Hepatology 2000;32:442-444.

[22] Fraschini F, Dermartini G, Esposti D. Pharmacology of silymarin. Clin Drug Invest $2002 ; 22: 51-65$.

[23] Radko L, Cybuski W. Application of sylimarin in human and animal medicine. J PreClin Clin Res 2007;1(1):22-26.

[24] Wisemann H. Dietary influences on membrane function: Importance in protection against oxidative damage and disease. J Nutr Biochem 1996;7:2-5.

[25] Sonnenbichler J, Zetl I. Biochemical effects of the flavonolignane silibinin on RNA, protein and DNA synthesis in rat livers. Prog Clin Biol Res 1986;213:319-331.

[26] Lahiri-Chatterjee M, Katiyar SK, Mohan RR, Agarwal R. A flavonoid antioxidant silymarin affords exceptionally high protection against tumor promotion in the SENCAR mouse skin tumorigenesis model. Cancer Res 1999;59:622-632.

[27] Katiyar SK, Lundgren DW, Mukhtar H, Agarwal R. Inhibitory effect of silymarin, an anti-hepatotoxic flavonoid, on 12-O-tetradecanoylphorbol-13-acetate-induced epidermal ornitine decarboxylase activity and mRNA in SENCAR mice. Carcinog 1994;15:1099-1103.

[28] Zhao J, Sharma Y, Agarwal R. Flavonoid antioxidant, silymarin, affords significant inhibition against 12-O-tetradecanoylphorbol 13-acetate-caused modulation of antioxidant and inflammatory enzymes, and cyclooxygenase 2 and interleukin-1a expression in SENCAR mouse epidermis: implications in the prevention of stage I tumor. Mol Carcinog 1999;26:321-333.

[29] Zhao J, Lahiri-Chatterjee M, Sharma Y, Agarwal R. Inhibitory effect of a flavonoid antioxidant silymarin on benzoyl peroxide-induced tumor promotion, oxidative stress 
and inflammatory responses in SENCAR mouse skin. Carcinog 2000;21:811-816.

[30] Bhatia N, Agarwal R. Detrimental effect of cancer preventive phytochemicals silymarin, genistenin and epigallocatechin 3-galate on epigenetic events in human prostate carcinoma DU145 cells. The Prostate 2001;46:98-107.

[31] Kuki Á, Nagy L, Deák Gy, Nagy M, Zsuga M, Kéki S. Identification of Silymarin Constituents : An Improved HPLC - MS Method. Chromatographia 2012;75:175180.

[32] Pyszková M, Biler M, Biedermann D, Valentová K, Kuzma M, Vrba J, Vacek J. Flavonolignan 2,3-dehydroderivatives: Preparation, antiradical and cytoprotective activity. Free Radic Biol Med 2016;90:114-125.

[33] Jovanovic SV, Steenken S, Tosic M, Marjanovic B, Simic MG. Flavonoids as Antioxidants. J Am Chem Soc 1994;116:4846-4851.

[34] Jovanovic SV, Steenken S, Hara Y, Simic MG. Reduction potentials of flavonoid and model phenoxyl radicals. Which ring in flavonoids is responsible for antioxidant activity? J Chem Soc Perkin Trans 2 1996;2:2497-2504.

[35] Litwinienko G, Ingold KU. Abnormal solvent effects on hydrogen atom abstraction. 1. The reactions of phenols with 2,2-diphenyl-1-picrylhydrazyl (dpph*) in alcohols. J Org Chem 2003;68(9):3433-3438.

[36] Zhang HY, Ji HF. How vitamin E scavenges DPPH radicals in polar protic media. New J Chem 2006;30:503-504.

[37] Anouar E, Kosinova P, Kozlowski D, Mokrini R, Duroux JL, Trouillas P. New aspects of the antioxidant properties of phenolic acids: a combined theoretical and experimental approach. Phys Chem Chem Phys 2009;11:7659-7668.

[38] Burton GW, Doba T, Gabe EJ, Hughes L, Lee FL, Prasad L, Ingold KU. Autooxidation of biological molecules. 4. Maximizing the antioxidant activity of 
phenols. J Am Chem Soc 1985;107:7053-7065.

[39] de Heer MI, Mulder P, Korth HG, Ingold KU, Lusztyk J. Hydrogen atom abstraction kinetics from intramolecularly hydrogen bonded ubiquinol-0 and other (poly)methoxy phenols. J Am Chem Soc 2000;122:2355-2360.

[40] Osorio E, Pérez EG, Areche C, Ruiz LM, Cassels BK, Flórez E, Tiznado W. Why is quercetin a better antioxidant than taxifolin? Theoretical study of mechanisms involving activated forms. J Mol Model 2013;19(5):2165-2172.

[41] Moss S, Zweifel H. Degradation and stabilization of high density polyethylene during multiple extrusions. Polym Degrad Stab 1989;25:217-245.

[42] Klemchuk PP, Horng PL. Transformation products of hindered phenolic antioxidants and colour developments in polyolefins. Polym Degrad Stab 1991;34:333-346.

[43] Breese KD, Lamethe JF, DeArmitt C. Improving synthetic hindered phenol antioxidants: learning from vitamin E. Polym Degrad Stab 2000;70:89-96. 